\title{
Blood-borne infections in Dublin's opiate users
}

\author{
M Fitzgerald ${ }^{1}$, J Barry ${ }^{2,3}$, P O'Sullivan ${ }^{3}$, L Thornton ${ }^{3}$ \\ Food Safety Authority of Ireland ${ }^{1}$, Lower Abbey Street, Dublin, Department of Community Health and \\ General Practice ${ }^{2}$, Trinity College, Dublin, and Department of Public Health ${ }^{3}$, ERHA, Dr Steevens' \\ Hospital, Dublin, Ireland.
}

\begin{abstract}
Background Injecting drug users are at high risk of acquiring blood-borne infections. Ireland has had a harm reduction policy of methadone maintenance and needle exchange since 1992.

Aim To estimate prevalence of hepatitis B, hepatitis C and HIV infection and appropriate uptake of hepatitis $\mathrm{B}$ vaccine in methadone attendees and to make recommendations for a simple record-based surveillance system.

Method Retrospective study of 138 client records for evidence of laboratory tests or test results for bloodborne viruses and appropriate immunisation against hepatitis $\mathrm{B}$.

Results A total of $60 \%$ of clients had evidence of one or more laboratory tests in their notes. Of those tested for individual viruses, $5.1 \%$ were positive for hepatitis B surface antigen, $78.8 \%$ had antibodies to hepatitis $\mathrm{C}$ and $16.7 \%$ were HIV positive. Nearly two-thirds of clients had no evidence of vaccination or information on prior immunity in their records.

Conclusions A standardised written protocol for screening for blood-borne viruses and for immunisation against hepatitis B in methadone service attendees was clearly needed, and was subsequently introduced by the Eastern Region Health Authority.
\end{abstract}

\section{Introduction}

Injecting drug users are known to be at high risk of acquiring blood-borne infections. ${ }^{1-3}$ Since 1992, the Irish Government has actively pursued a policy of harm reduction in injecting drug users by providing methadone maintenance and needle exchange. ${ }^{4-6}$ This resulted in an increase in the number of opiate users in the Eastern Health Board (EHB) region in receipt of methadone from 150 in 1992 to just under 3,000 by the end of 1997 ( 715 of whom were attending methadone dispensing clinics run by the Health Board).

However, information relating to the prevalence in these opiate users of the blood-borne diseases, hepatitis B (HBV), hepatitis C (HCV) and human immunodeficiency virus (HIV), had not been comprehensively documented at that time, although reports were later published on these infections in specific sub-groups of opiate users in 1998. ${ }^{7,8}$ When this survey was undertaken at the end of 1997 there was no simple means of ascertaining the prevalence of bloodborne infections in this client group.

The aims of this study were as follows: to determine the prevalence of HBV, HCV and HIV in opiate users attending methadone clinics in the EHB region based on information already available in their clinical records; to determine the proportion of susceptible opiate users in this population immunised against $\mathrm{HBV}$; and to make recommendations on a simple surveillance system to enable easier access to prevalence data in the EHB Drugs/AIDS Services.

\section{Method}

The study population was 715 opiate users who were attending five dedicated EHB run methadone dispensing clinics in December 1997. Following a statistical analysis based on the expected prevalence of bloodborne infections in this population, it was decided that taking a randomly selected $20 \%$ sample would be adequate to achieve a significant result. ${ }^{1,2}$ To allow for initial interview and history taking of new clients, only those in the service for at least four weeks were included in the survey. Each clinical record was examined on-site in the clinics by one of the authors (MF) for a record of the blood-borne infections, either in the form of laboratory reports or in the hand-written clinical notes and letters. Documented information on hepatitis B vaccination status was also looked for in the record. Data were entered and analysed using Epi Info version 6.04b. ${ }^{9}$

Hepatitis B virus screening and immunisation in people who are not immune should ideally be commenced within one month of starting methadone and the course of three doses completed within the next six months. Therefore, in order to give a realistic estimate of hepatitis B vaccination uptake, evidence ofimmunisation was only looked for in clients who had been attending a methadone dispensing clinic for at least six months at the time of the study.

\section{Results}

The records of 143 clients were examined, of whom 138 had been attending the service for more than four weeks. 
Ninety-nine $(72 \%)$ of the sample were male. The mean age was 29 years $(\mathrm{SD} \pm 6.7)$ and $45(32.6 \%)$ were under 25 years. The median duration of attendance in the methadone service was eight months. Eighty-three $(60 \%)$ had a laboratory test result for blood-borne viruses in the notes. The estimated prevalence of the infections, based on laboratory reports seen and a combination of those laboratory reports and the handwritten medical notes, is given in Table 1.

Evidence of hepatitis B vaccination was only looked for in the records of the 85 clients in the sample who had been attending a clinic for at least six months (see Table 2).

Table 1. Blood-borne viral infections in a random sample of clients attending EHB methadone clinics in December 1997

\begin{tabular}{|c|c|c|}
\hline \multirow[t]{2}{*}{$\begin{array}{l}\text { Viral marker } \\
\text { (Number who had test) }\end{array}$} & \multicolumn{2}{|c|}{$\begin{array}{l}\text { Prevalence in those } \\
\text { with reported results* }\end{array}$} \\
\hline & $\begin{array}{l}\text { Test } \\
\text { No. }\end{array}$ & $\begin{array}{c}\text { Positive } \\
\%\end{array}$ \\
\hline Hepatitis B surface antigen $(n=79)$ & 4 & 5.1 \\
\hline Hepatitis B core antibody $(n=64)$ & 18 & 28.1 \\
\hline Hepatitis $\mathrm{C}$ antibody $(\mathrm{n}=99)$ & 78 & 78.8 \\
\hline HIV antibody $(n=90)$ & 15 & 16.7 \\
\hline
\end{tabular}

Table 2. Hepatitis B vaccination status (as documented in medical records) in December 1997 in 85 clients attending EHB methadone clinics*

\begin{tabular}{lcc}
\hline Description & Number & $\mathbf{\%}$ \\
\hline $\begin{array}{l}\text { Evidence of past infection } \\
\text { (Hepatitis: B core-antibody +ve) }\end{array}$ & 15 & 7.6 \\
$\begin{array}{l}\text { Partially immunized (1-2 doses } \\
\text { of hepatitis B vaccine given) }\end{array}$ & 2 & 2.4 \\
$\begin{array}{l}\text { Fully immunized (3 doses of } \\
\text { hepatitis B vaccine given) }\end{array}$ & 13 & 15.3 \\
$\begin{array}{l}\text { Not vaccinated or information } \\
\text { on immunity not available }\end{array}$ & 55 & 64.7 \\
Total & & \\
*For six months or more & $\mathbf{8 5}$ & $\mathbf{9 0}$ \\
\hline
\end{tabular}

\section{Discussion}

This study was carried out in a multidisciplinary specialist setting of the health board clinics. These clinics are staffed by GPs on a sessional basis, with paramedical and clerical support — the primary aim being to support methadone replacement. A low level of comprehensively documented serological testing for blood-borne infections was found and it was difficult to establish the prevalence of these diseases accurately. Reasons ascertained from the notes for this low level of testing included the short time most clients were in the service, their high turnover and their mobility due to prison sentences and inpatient hospital care. Health workers pointed out the difficulties in carrying out blood tests when pre-test counselling was required, as this often meant the client returning on another day with the potential for defaulting.

Examination of medical records revealed that clients were also less likely to have had a blood test if they were described on first interview as "not sharing needles" or "not using needles". There were no agreed standard procedures for screening of blood-borne viruses in use at the clinics. This highlighted the need for a standardised protocol for screening and immunisation.
The standards of medical records varied between centres, and patients' notes were incomplete in some centres. The level of clerical support also varied between centres and this may have been reflected in the difficulties in maintenance of detailed and complex records. An audit of the service such as this highlights the difficulties in keeping medical records for a mobile population in a primary care setting. Clients attending the main methadone dispensing clinics tend to be the most chaotic drug users in the system.

The prevalence of antibodies to hepatitis B core antigen in patients known to have had the test in this study was $28.1 \%$, indicating the high level of exposure in this group. This is considerably higher than that of prisoners who were injecting drugs in another recent study of the prevalence of blood-borne infections $(18.5 \%){ }^{10}$

HCV seroprevalence is very high in this study (78.8\%), although those under 25 years of age had lower recorded prevalence rates (52\%). The overall prevalence rate is higher than that described by Smyth et al in shortterm injecting drug users in Dublin between 1993 and $1996(52.1 \%)^{7}$ and slightly lower than the figure found by Allwright et al in drug using Irish prisoners in 1998 where more than one-third of all prisoners and $80 \%$ of drug using prisoners were positive for antibodies to hepatitis $\mathrm{C}$ virus. ${ }^{10}$ According to their records, clients in our study had generally been using drugs over a longer duration than the drug users in the study by Smyth et al. ${ }^{7}$ The fact that there was no surveillance information "for HCV" on a significant percentage $(28 \%)$ of the study population may have also resulted in a higher prevalence of $\mathrm{HCV}$, since the clients who have no record of a test result might have been those perceived to be less at risk or who had tested negative. This desk-based study, therefore, has limitations in estimating prevalence rates, as possibly those who were most likely to have evidence of a test were those with greatest risk of being positive.

Estimated prevalence rates for HIV infection in this group $(16.7 \%)$ were higher than those found by Dorman et al in $1997^{3}(8.4 \%)$ in drug users, and also higher than those found by Allwright ${ }^{10}$ in drug users in Irish prisons $(3.5 \%)$. However, as with $\mathrm{HCV}$, no one under 25 years was positive for HIV in the study group, indicating the potential for a positive impact of harm reduction strategies in younger addicts.

Evidence of uptake of hepatitis B vaccination in the study group was poor. Even though short-term attendees $(n=53)$ were excluded from this part of the analysis, only one-third of the remaining 85 clients had evidence of either immunity, or partial or full immunisation. The remaining two-thirds had either not been vaccinated or did not have any record in their notes to indicate that they had been vaccinated. Evidence of a test to ascertain anti-HBs status after vaccination, as recommended by vaccine protocol for the methadone service, was also not present in most of the records. Vaccine refusals were not recorded.

While this study was primarily an audit of screening for infectious diseases and immunisation practice, it is of limited value in estimating true prevalence rates of blood-borne viruses and of practices in immunisation. Despite this, the study highlights the 
need for a written standard protocol for screening and immunisation in attendees at methadone programmes.

High levels of $\mathrm{HCV}$ infection reflect the infectivity of this virus and the need for early intervention in injecting drug users. ${ }^{11}$ With regard to $\mathrm{HBV}$, there is also scope for improve-

ment in terms of offering screening and immunisation to susceptible drug users and their contacts as early as possible, with good documentation to flag defaulters to other health professionals and outreach workers. ${ }^{12}$

Provision of an accurate surveillance system for these infections in this population is complex. The logistics of carrying out HTV surveillance in a less complex population have been documented. ${ }^{13}$ Following this study, in consultation with the doctors working in the ERHA's Drugs Service, a standard protocol was developed for screening their patients for blood-borne infectious diseases; it was introduced in October 1998. At the same time, a front sheet was developed to go in each patient's records to summarise his or her status regarding the screening for blood-borne infections, the results of the tests for the various antigens and immunisation.

The overall aims of implementing this standard protocol throughout tile Drugs Service are to improve the screening for blood-borne diseases of drug users entering the methadone service and to ensure that the results of that screening are recorded and then acted upon, where appropriate. The protocol proposes that all 'rug users receiving methadone in the ERHA's Drugs Service should be offered screening for hepatitis B, hepatitis $\mathrm{C}$ and HIV within four weeks of commencing on a methadone programme. If a patient refuses screening for one or all of the viruses, it should be clearly recorded in the notes giving the reason. The tests for hepatitis C and HIV should be repeated annually so long as the patient remains negative for these viruses. Hepatitis B testing should be done using two serological markers, HBsAg and anti-core antibody. If neither of these markers is positive, a course of immunisation against hepatitis B infection should be offered. Refusals should again be recorded. The first dose of immunisation should be offered when the negative screening results are available with boosters to be given at one month and at six months.

This protocol has now been implemented throughout the ERHA's Drugs Service and a study is in progress to audit its implementation.

\section{References}

1. Madden PB, Lamagni T, Hope V. The HIV epidemic in injecting drug users. Commun Dis Rep 1997; 7: 128-30.
2. Bolumar F, Hernandez-Aguado I, Ferrer L. Prevalence of antibodies to hepatitis $\mathrm{C}$ in a population of intravenous drug users in Valencia, Spain, 19901992. Int J Epidemiol 1996; 25: 204-9.

3. Dorman A, Keenan E, Schuttler C et al. HIV risk behaviour in Irish intravenous drug users. Ir $J$ Med Sci 1997; 166(4): 235-8.

4. Department of Health (Ireland) Government Strategy to Prevent Drug Misuse. May 1991.

5. Dean G, Kelly M, O'Hare A, Kelly G, O'Connor A. The opiate epidemic in Dublin 19781983. Ir Med J 1985; 78(4)- 107,10.

6. Butler S. Drug problems and drug policies in Ireland. A quarter of a century review. Administration 1991; 39: 210-33.

7. Smyth BP, Keenan E, O'Connor JJ. Evaluation of the impact of Dublin's expanded harm reduction programme on prevalence of hepatitis $\mathrm{C}$ amongst short-term injecting drug users with short term injecting histories. J Epidemiol Community Health 1999; 53: 434-5.

8. Cassin S, Geoghegan T. Young injectors: a comparative .1 analysis of risk behaviour. Ir J Med Sa 1998; 167(4): 234-7.

9. Dean AG, Dean JA, Coulombier D et al. Epi Info, Version 6; a word processing, database and statistics programme for epidemiology on microcomputers. Centers for Disease Control and Prevention, Atlanta, Georgia, USA, 1994.

10. Allwright S, Bradley F, Long J et al. Prevalence of anti-bodies to hepatitis B, hepatitis C and HIV and risk factors in Irish prisons: results of a national crosssectoral survey. Br Med J 2000; 321: 78-82.

11. Stark K, Mueller R, Bienzle U. Frontloading: a risk factor for HIV and hepatitis $\mathrm{C}$ virus infection among injecting drug users in Berlin. AIDS 1996; 10: 311-7.

12. Crofts N, Campbell K. Incidence of blood-borne virus infection and risk behaviours in a cohort of injecting drug users in Victoria, 1990-1995. Med $J$ Aust 1997; 167: 17-9.

13. Schwartlander B, Janssen RS, Satten G. Guidelines for designing rapid assessment surveys of HIV seroprevalence among hospitalised patients. Public Health Rep 1994; 109: 53-9.

Correspondence to: Dr Margaret Fitzgerald, chief specialist, Public Health Food Safety Authority of Ireland, Abbey Court, Lower Abbey Street, Dublin 1, Ireland. Tel: +35318171300; fax: +3531878 6429. 